\title{
Co-expression analysis and ceRNA network reveal eight novel potential IncRNA biomarkers in hepatocellular carcinoma
}

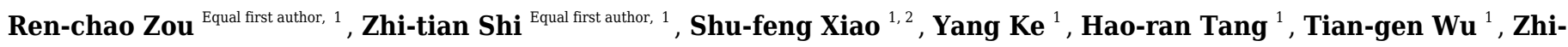 \\ tang Guo ${ }^{1}$, Fan Ni ${ }^{1}$, Sanqi An ${ }^{\text {Corresp., }}{ }^{3}$, Lin Wang ${ }^{\text {Corresp. } 1}$ \\ ${ }^{1}$ Department of Hepatobiliary Surgery, the Second Affiliated Hospital of Kunming Medical University, kunming, China \\ 2 Department of General Surgery, Puer People's Hospital, Puer, China \\ ${ }^{3}$ Center for Stem Cell Biology and Tissue Engineering, Key Laboratory of Ministry of Education, Sun Yat-Sen University, Guangzhou, China \\ Corresponding Authors: Sanqi An, Lin Wang \\ Email address: ansq@mail2.sysu.edu.cn, wanglinfey@126.com
}

Background: Hepatocellular carcinoma $(\mathrm{HCC})$ is the most common primary liver cancer in the world, with a high degree of malignancy and recurrence. The influence of ceRNA network in tumor on the biological function of liver cancer is very important, It has been reported that many IncRNA play key role in liver cancer development. In our study, integrated data analysis revealed potential eight novel IncRNA biomarkers in hepatocellular carcinoma. Methods: Transcriptome data and clinical data were downloaded from the The Cancer Genome Atlas ( TCGA ) data portal.Weighted gene co expression network analysis was performed to identify the expression pattern of genes in liver cancer. Then ceRNA network was constructed using transcriptome data. Results: The integrated analysis of miRNA and RNAseq in the database show eight novel IncRNAs that may be involved in important biological pathways including TNM and disease development in liver cancer. We performed function enrichment analysis about mRNAs affected by these IncRNAs. Conclusions: By identifying the ceRNA network and IncRNAs that affect liver cancer, we showed that eight novel IncRNAs played an important role in the development and progress of liver cancer. 
1 Co-expression analysis and ceRNA network reveal eight novel potential IncRNA biomarkers

2 in hepatocellular carcinoma

3

4 Ren-chao Zou ${ }^{1, \#}$, Zhi-tian Shi ${ }^{1, \#}$, Shu-feng Xiao ${ }^{1,2}$, Yang Ke ${ }^{1}$, Hao-ran Tang ${ }^{1}$, Tian-gen Wu ${ }^{1}$, Zhi5 tang $\mathrm{Guo}^{1}$, Fan $\mathrm{Ni}^{1}$, Sanqi $\mathrm{An}^{3, *}$, Lin Wang, ${ }^{1, *}$

$6{ }^{1}$ Department of Hepatobiliary Surgery, The Second Affiliated Hospital of Kunming Medical 7 University, Kunming, 650101, Yunnan, China.

$8 \quad 2$ Department of General Surgery, Puer People’s Hospital, Puer, 665000, Yunnan, China

$9{ }^{3}$ Center for Stem Cell Biology and Tissue Engineering, Key Laboratory of Ministry of Education,

10 Sun Yat-sen University, Guangzhou 510080, Guangdong, China

11 \# These authors contributed equally to this article as co-first authors.

$12 *$ Corresponding Author:

13 Sanqi An (ansq@,mail2.sysu.edu.cn)

14 Lin Wang (wanglinfey@,126.com)

15 Tel.: +86-871-65352087

16 Fax: $+86-871-65352087$

Background: Hepatocellular carcinoma (HCC) is the most common primary liver cancer in the world, with a high degree of malignancy and recurrence. The influence of ceRNA network in tumor on the biological function of liver cancer is very important, It has been reported that many lncRNAs play key role in liver cancer development. In our study, integrated data analysis reveal eight novel potential lncRNA biomarkers in hepatocellular carcinoma.

Methods: Transcriptome data and clinical data were downloaded from the The Cancer Genome Atlas (TCGA) data portal. Weighted gene co-expression network analysis was performed to identify the expression pattern of genes in liver cancer. Then ceRNA network was constructed using transcriptome data. 
27 Results: The integrated analysis of miRNA and RNAseq in the database show eight novel lncRNAs that may be involved in important biological pathways including TNM and disease development in liver cancer. We performed function enrichment analysis about mRNAs affected by these lncRNAs.

Conclusions: By identifying the ceRNA network and lncRNAs that affect liver cancer, we showed that eight novel lncRNAs played an important role in the development and progress of liver cancer.

\section{Introduction}

Liver cancer, most common among male patients, is the high leading cause of death (Wang et al. 2015). In the United States, there are as many as 42,030 new cases and 31,780 deaths related to liver cancer every year, based on the latest statistics record (Siegel et al. 2019). The degree of malignancy of cancer can be determined by study of the histology of the tumor, and patients can be divided into three classes, low, medium, and high according to the degree of malignancy. Clinically, primary liver carcinoma is considered to be one of the most common malignant tumors, and about $90 \%$ of these tumors are hepatocellular carcinoma (HCC). In cases of patients diagnosed with HCC, $50.7 \%$ of them achieve a 5-year survival rate, while the median survival time is 60 months (Lee et al. 2006). The prognosis of HCC patients is related to the patient's disease stage. Currently, the tumor-node-metastasis (TNM) pathological staging standard developed by the American Joint Committee on Cancer (AJCC) is the most commonly used malignant tumor staging system worldwide. However, the relationship between long non-coding RNAs (lncRNAs) and tumor staging has raised concern among researchers (Chen et al. 2015; Ou et al. 2018; Yao et al. 2018), suggesting that many lncRNAs play progressive key roles in HCC development (Chen et al. 2016a). For example, the MALAT-1 gene is upregulated in HCC and also correlates with prognostics and recurrence (Guerrieri 2015; Lai et al. 2012); the overexpression of the HULC gene may reduce the mir-372 gene, while at the same time may promote reprogramming during tumorigenesis (Du et al. 2012); and DANCR induces stemness features and could serve as a potential prognostic marker and therapeutic target for HCC (Yuan et al. 2016). Previous studies have mainly focused on the single biomarker use of miRNAs in HCC.

The weighted gene co-expression network analysis (WGCNA) is a popular bioinformatic method used in the construction of gene networks and the detection of gene modules and their phenotypic traits (Langfelder \& Horvath 2008; Yin et al. 2018). In this study, we identified eight novel lncRNAs correlated with TNM using WGCNA. Additionally, functional enrichment analysis shows that these eight novel lncRNAs play an important role in the regulation of gene expression that affect development and progression of liver cancer.

\section{Materials and Methods}




\subsection{Data preprocessing and differential gene selection}

62

The clinical data, RNAseq data and microRNA data of LIHC were downloaded from the The Cancer Genome Atlas (TCGA) database (Akbani et al. 2014) (Table S1). There were 49 pairs of microRNA samples and 50 pairs of RNAseq samples in total. The variation between the RNA and microRNAs data was calculated using EdgeR package (Reimers \& Carey 2006; Varet et al. 2016). Only microRNAs recorded with first 10 and last $10 \mathrm{FC}$ values were selected for subsequent analysis(Li et al. 2018; Shao \& Li 2019). Because these biggest expression changed miRNA may have real major function in HCC. For RNAseq data, the EdgeR package was used to calculate the differential correlationship, and the threshold value for FDR was set at $<0.01,|\log F C|>1$ (Table S2). Clinical data were used to calculate the correlation matrix of clinical information for integrated analysis. We used the "heatmap.2" function in the "gplots" package to create the heatmap.

\subsection{Determination of ceRNA}

Using microRNAs and differential expression genes as input data for target prediction, the RNA22 program was used to predict binding sites of microRNAs based on their sequence characteristics (Loher \& Rigoutsos 2012). Based on these ceRNA interactions, we obtained all the mRNA-miRNA pairs with sharing the number of common miRNAs. Because the number of the ceRNA pairs is obey hypergeometric distribution. We estimated their statistical significance by a hypergeometric test. The potential top 20 different expression miRNA with hypergeometric test in ceRNA network P value less than 0.05 was obtained (Li et al. 2018; Shao \& Li 2019). Specific formulas, such as the differential expression matrix of lncRNA, were used to get the Pearson correlation coefficient (PCC) (Table S3). Based on ceRNA's mechanism, the expression matrix (EM) for IncRNA to bind between themselves resulted in a PCC of EM $>0$. Co-expression is one of the features of ceRNA network on account of their interactions. The final ceRNA pair was obtained by intersecting the ceRNA threshold with cutoff $p$-value $<0.05$. Cytoscape v3.0 was used to construct the ceRNA network. The overall Kaplan-Meier (KM) survival analysis in each subtype was performed using GEPIA2 database (Tang, 2017).

\subsection{WGCNA model related computing}

Weighted gene co-expression network analysis (WGCNA), a bioinformatic method used to find correlation patterns among genes, was utilized in this research. WGCNA assumes that gene expression networks is scale-free, it uses a 'soft' threshold to determine the weights of the edges connecting genes and merge individual genes to a module. An appropriate soft threshold will make 
93 the co-expression network closer to a scale-free network. Then we constructed a signed weighted co-expression network using WGCNA based on the gene expression value across the TCGA samples. We obtained 60 co-expression modules according to the correlations of fpkm value among samples. Each module is represented by an value belongs to the 'eigengene'. This value is identified from the principal component analysis (PCA) of all the gene expression value in the module. Then, we find the relationship between modules and the trait, Eventually, unsupervised WGCNA identified major lncRNAs expression modules with different degrees of correlation to TNM staging using WGCNA R software (Langfelder \& Horvath 2008).

\subsection{Prediction of interrelationship between IncRNA-related mRNAs}

Using RNAseq data from TCGA, the correlation prediction of the lncRNA-lncRNA network and lncRNA-mRNA network was constructed. The cutoff of PCC was 0.7. We retained the intersection with the lncRNA using a cutoff of the top $20 \%$ degree. Ultimately, we found eight potentially important lncRNAs with high degrees of connection in the ceRNA network and high correlation at the expression level.

\subsection{Gene Ontology and KEGG Enrichment Analysis}

Gene ontology (GO) annotation analysis was performed using DAVID software (Huang da et al. 2009a). Gene functions for these important indirectly regulated mRNA genes elucidate that this mRNA regulated by lncRNA may have some key biological functions (Huang da et al. 2009a; Huang da et al. 2009b). String database was used for protein-protein analysis(Szklarczyk et al. 2017).

\section{Results}

\subsection{Analysis of differential miRNAs and differential IncRNAs}

The differential expression of miRNAs and lncRNAs between normal samples and cancer samples was calculated separately using the EdgeR package (Chen et al. 2017; Law et al. 2016; Maza 2016). There were 1962 significant differential expression genes in lncRNAs, and 310 differentially expressed miRNA genes found between healthy and cancer-treated samples. Almost half of the mRNAs are upregulated and half downregulated (Fig.1A). Most of the gene expression for lncRNAs and miRNAs was upregulated in cancer-treated samples (Fig.1B-C).

\subsection{Weighted gene co-expression network analysis of IncRNA}


122 To determine if any of the identified coexpression modules were associated with TNM stage, we

123

124

125

126

127

128

129

130

131

132

133

134

135

136

137 calculated the PCC between the MEs and TNM stage. All lncRNAs were merged to 60 modules according the degree of coexpression across the data set in WGCNA. As in the previous study, we assigned each coexpression module an arbitrary color for reference(Di et al. 2019; Zhussupbekova et al. 2016). The hierarchical clustering dendrogram of the eigengenes shows the module size (the number of genes per module ) and relationships among these modules (Fig.2A). Most modules had minimal relationships with each other.

Comparison of module-characteristic eigengenes showed TNM was best correlated with the module MElightpink4 ( $\mathrm{p}=4 \mathrm{E}-04)$ and MElightcyan $(\mathrm{P}<0.006)$, composed of 125 lncRNAs (Fig. 2B). The PCC values ranged from -1 to +1 depending on the power of the relationship. A positive value indicated that the lncRNA within a particular co-expression module increased as the TNM increased, whereas the opposite occurred if the sign of the PCC was negative. We learned that the correlationship between the module and TNM stage with PCC value was accompanied by the corresponding $\mathrm{P}$-value in brackets. These modules included genes that were co-expressed in a particular TNM stage can be used to represent the TNM stage of HCC development (Fig. 2B). These gene may be the risk factors and therapeutic targets in the treatment of HCC.

\section{3. ceRNA network of IncRNA reveals potential biomarkers in liver cancer}

As shown in Figure 3A, ceRNA network was constructed by high degree lncRNAs. The topological characteristics of ceRNA network were analyzed with degree, betweenness, and closeness (Table S4). Many genes in ceRNA network are with high degree, betweenness, or closeness like AC016773.1, AC145285.2, LINC01569 and DANCR, and so on. This implied ceRNA network may regulate many gene expression through these lncRNAs to have effect on progression of liver cancer. To verify the function of these lncRNAs, Kaplan-Meier survival analysis was perform ed for expression level of these lncRNAs. The results of the survival analysis presented in Figure 3B and Figure $3 \mathrm{C}$ show patients with high expression level of DANCR or AL671710.1 have poor prognosis. It is a validation of our analysis and two of the lncRNAs may affect prognosis.

\subsection{The interactions between IncRNAs and mRNAs}

Combining WGCNA with the results of ceRNA prediction analysis, lncRNA was combined with correlation prediction to find eight important lncRNAs including AL671710.1, TRIM52-AS1, C1orf220, AC022762.2, DANCR, LINC01569, AC084018.1 and MIR194-2HG. Among these IncRNAs, downregulation of TRIM52-AS1 play key role in renal cell carcinoma(Liu et al. 2016). DANCR increases stemness features of hepatocellular carcinoma (Yuan et al. 2016). DANCR is also associated with various cancer(Lu et al. 2018a; Lu et al. 2018b; Mao et al. 2017; Sha et al. 
156

157

158

159

160

161

162

163

164

165

166

167

168

169

170

171

172

173

174

175

176

177

178

179

180

181

182

183

184

185

186

187

188

189

2017; Xu et al. 2018; Yuan et al. 2016). However, there is little known about the function of other six lncRNAs in cancer. There were 124 genes targeted by these eight lncRNAs (Fig.4A-H), including EIF3 which functions during the initiation phase of translation. TRIM52-AS1 may influence cancer behavior and function through interactions with regulator EIF3. EIF3 plays a key role in human diseases (Gomes-Duarte et al. 2018; Valasek et al. 2017). Also, there were 30 genes targeted by lncRNA ac084018.1, including m6A reader methyltransferase like 3 (METTL3). As reported in previous research, METTL3 promotes liver cancer progression through YTHDF2 (Balacco \& Soller 2019; Berlivet et al. 2019; Chen et al. 2018; Weng et al. 2018). DANCR and AL671710.1 also have crucial roles through certain key genes (Fig.4C,E).

\subsection{GO and KEGG pathway enrichment analyses of IncRNA-targeted gene}

Additionally, we performed GO and KEGG enrichment analysis of the mRNAs in the network (Fig.5A-C). We analysed target genes of the lncRNA based on their enrichment scores for associated GO terms and KEGG pathways using David tools (Huang da et al. 2009a; Huang da et al. 2009b). The GO and KEGG enrichment analysis concerning the target genes of lncRNAs indicated that the top regulated pathways of IncRNAs were Huntington's disease, RNA polymerase and Pyrimidine metabolism, and the top regulated functions of lncRNAs were SRP-dependent cotranslational protein targeting to membrane, translational initiation, viral transcription, nucleartranscribed mRNA catabolic process, and nonsense-mediated decay. Further-more, those essential mRNA may interact with each other and function in HCC (Fig. 6A). We can conclude that those lncRNAs affect HCC through the functions and pathways listed above (the flow chart was depicted in Fig. 6B).

\section{Discussion}

Hepatocellular carcinoma (HCC) is one of the primary causes of cancer-related death worldwide (Balogh et al. 2016). Many genes influence HCC, TP53 tumor-suppressor gene, p16INK4A and $\mathrm{Rb}$-associated with various risk factors have been largely reported (Bae et al. 2016; Buendia 2000; Nishida \& Fukuda 2001; Peng et al. 2013; Wang et al. 2017; Zhang et al. 2014). Some even take part in the cancer biography progress through lncRNA ( $\mathrm{Su}$ et al. 2017). Long non-coding RNAs (lncRNAs) which are transcribed but do not encode proteins, play key roles in HCC development (Abbastabar et al. 2018). These include MALAT-1 and also NEAT-2, which regulates splicing factors mostly situated in nuclear speckles. In addition, MALAT-1 is a biomarker in various cancers including HCC(Lai et al. 2012; Wang et al. 2016). LncRNA GAS5 is a biomarker and have potential applications in HCC therapy(Fang et al. 2019). Using WGCNA and hypergeometric test analysis, we found eight lncRNAs with important functions: AL671710.1, TRIM52-AS1, C1orf220, AC022762.2, DANCR, LINC01569, AC084018.1 and MIR194-2HG. TRIM52-AS1 is 
one of the eight lncRNAs that has been reported as a function of a tumor suppressor (Liu et al. 2016; Zhang et al. 2017). Targeted by MYC, DANCR promotes cancer (Chen et al. 2016b; Dhanasekaran et al. 2017; Huang et al. 2013; Kron et al. 2012; Lu et al. 2018b). Taken together, we concluded that these lncRNAs may function as a potential tumor regulator in HCC. Additionally, some lncRNAs were associated with the TNM stage in HCC tissues (Abbastabar et al. 2018). The American Joint Committee on Cancer (AJCC) stratifies patients using a TumorNode-Metastasis (TNM) classification, representing a group of models useful in the assessment of tumor extension (Selcuk 2017; Tellapuri et al. 2018). Among several staging systems, the TNM system was one of the most widely accepted, and had a higher prognostic competency than the other systems (Prognosis Evaluation in Patients with Hepatocellular Carcinoma after Hepatectomy, Comparison of BCLC and Hangzhou Criteria Staging Systems). In our work, we found two lncRNAs modules associated with the TNM stage. Those lncRNAs may function as the biomarker of node size and metastasis status in HCC. Systematic analysis of transcriptomics data reveal those novel potential therapeutic target may be involved in cancer-related pathway in liver cancer. Our study has limitations, the specific mechanism of these lncRNAs remains unexplored.

\section{Conclusions}

In summary, our results demonstrated that lncRNAs AL671710.1, TRIM52-AS1, C1orf220, AC022762.2, DANCR, LINC01569, AC084018.1, and MIR194-2HG play an essential role in the HCC stage, and their targeted mRNA have key functions in HCC. Those lncRNAs might be a novel prognostic biomarker for HCC.

\section{References}

Abbastabar M, Sarfi M, Golestani A, and Khalili E. 2018. lncRNA involvement in hepatocellular carcinoma metastasis and prognosis. EXCLI J 17:900-913. 10.17179/excli2018-1541

Akbani R, Ng PK, Werner HM, Shahmoradgoli M, Zhang F, Ju Z, Liu W, Yang JY, Yoshihara K, Li J, Ling S, Seviour EG, Ram PT, Minna JD, Diao L, Tong P, Heymach JV, Hill SM, Dondelinger F, Stadler N, Byers LA, Meric-Bernstam F, Weinstein JN, Broom BM, Verhaak RG, Liang H, Mukherjee S, Lu Y, and Mills GB. 2014. A pan-cancer proteomic perspective on The Cancer Genome Atlas. Nat Commun 5:3887. 10.1038/ncomms4887

Bae JS, Noh SJ, Kim KM, Jang KY, Park HS, Chung MJ, Park BH, and Moon WS. 2016. PIN1 in hepatocellular carcinoma is associated with TP53 gene status. Oncol Rep 36:24052411. 10.3892/or.2016.5001

Balacco DL, and Soller M. 2019. The m(6)A Writer: Rise of a Machine for Growing Tasks. Biochemistry 58:363-378. 10.1021/acs.biochem.8b01166 
224

225

226

227

228

229

230

231

232

233

234

235

236

237

238

239

240

241

242

243

244

245

246

247

248

249

250

251

252

253

254

255

256

257

258

259

260

261

262

263

264

Balogh J, Victor D, 3rd, Asham EH, Burroughs SG, Boktour M, Saharia A, Li X, Ghobrial RM, and Monsour HP, Jr. 2016. Hepatocellular carcinoma: a review. J Hepatocell Carcinoma 3:41-53. 10.2147/JHC.S61146

Berlivet S, Scutenaire J, Deragon JM, and Bousquet-Antonelli C. 2019. Readers of the m(6)A epitranscriptomic code. Biochim Biophys Acta Gene Regul Mech. 10.1016/j.bbagrm.2018.12.008

Buendia MA. 2000. Genetics of hepatocellular carcinoma. Semin Cancer Biol 10:185-200. 10.1006/scbi.2000.0319

Chen D, Sun Q, Cheng XF, Zhang LF, Song W, Zhou DK, Lin JJ, and Wang WL. 2016a. Genome-wide analysis of long noncoding RNA (lncRNA) expression in colorectal cancer tissues from patients with liver metastasis. Cancer Medicine 5:1629-1639. 10.1002/cam4.738

Chen MN, Wei L, Law CT, Tsang FHC, Shen JL, Cheng CLH, Tsang LH, Ho DWH, Chiu DKC, Lee JMF, Wong CCL, Ng IOL, and Wong CM. 2018. RNA N6-methyladenosine methyltransferase-like 3 promotes liver cancer progression through YTHDF2-dependent posttranscriptional silencing of SOCS2. Hepatology 67:2254-2270. 10.1002/hep.29683

Chen T, Xie W, Xie L, Sun Y, Zhang Y, Shen Z, Sha N, Xu H, Wu Z, Hu H, and Wu C. 2015. Expression of long noncoding RNA lncRNA-n336928 is correlated with tumor stage and grade and overall survival in bladder cancer. Biochem Biophys Res Commun 468:666670. 10.1016/j.bbrc.2015.11.013

Chen Y, Pal B, Visvader JE, and Smyth GK. 2017. Differential methylation analysis of reduced representation bisulfite sequencing experiments using edgeR. F1000Res 6:2055. 10.12688/f1000research.13196.2

Chen YM, Lin CZ, Liu Y, and Jiang Y. 2016b. HMGB1 promotes HCC progression partly by downregulating p21 via ERK/c-Myc pathway and upregulating MMP-2. Tumor Biology 37:4399-4408. 10.1007/s13277-015-4049-z

Dhanasekaran R, Baylot V, Mosley A, and Felsher D. 2017. MYC is the master switch between tumor dormancy and relapse in Hepatocellular carcinoma (HCC). Hepatology 66:966a966a.

Di Y, Chen D, Yu W, and Yan L. 2019. Bladder cancer stage-associated hub genes revealed by WGCNA co-expression network analysis. Hereditas 156:7. 10.1186/s41065-019-0083-y

Du Y, Kong G, You X, Zhang S, Zhang T, Gao Y, Ye L, and Zhang X. 2012. Elevation of highly up-regulated in liver cancer (HULC) by hepatitis B virus X protein promotes hepatoma cell proliferation via down-regulating p18. J Biol Chem 287:26302-26311. 10.1074/jbc.M112.342113

Fang P, Xiang L, Chen W, Li S, Huang S, Li J, Zhuge L, Jin L, Feng W, Chen Y, and Pan C. 2019. LncRNA GAS5 enhanced the killing effect of NK cell on liver cancer through regulating miR-544/RUNX3. Innate Immun 25:99-109. 10.1177/1753425919827632

Gomes-Duarte A, Lacerda R, Menezes J, and Romao L. 2018. eIF3: a factor for human health and disease. RNA Biol 15:26-34. 10.1080/15476286.2017.1391437

Guerrieri F. 2015. Long non-coding RNAs era in liver cancer. World J Hepatol 7:1971-1973.

PeerJ reviewing PDF | (2019:04:36373:2:1:NEW 4 Oct 2019) 
10.4254/wjh.v7.i16.1971

Huang da W, Sherman BT, and Lempicki RA. 2009a. Bioinformatics enrichment tools: paths toward the comprehensive functional analysis of large gene lists. Nucleic Acids Res 37:113. 10.1093/nar/gkn923

Huang da W, Sherman BT, and Lempicki RA. 2009b. Systematic and integrative analysis of large gene lists using DAVID bioinformatics resources. Nature Protocols 4:44-57. 10.1038/nprot.2008.211

Huang ZS, Deng ZH, and Zhou XH. 2013. Oxymatrine influence proliferation and expression of E2F1 and c-myc in HCC cell line Bel-7404. Journal of Gastroenterology and Hepatology 28:783-783.

Kron C, Wittke E, Mottar A, Bothwell P, Finger R, and Bode BP. 2012. Amino acid transporters ASCT2 and LAT1 ubiquitously function in N-myc $(+)$ epithelial and mesenchymal human HCC cells exhibiting a wide array of mTOR and glycolytic reliance for growth. Cancer Research 72. 10.1158/1538-7445.Am2012-5148

Lai MC, Yang Z, Zhou L, Zhu QQ, Xie HY, Zhang F, Wu LM, Chen LM, and Zheng SS. 2012. Long non-coding RNA MALAT-1 overexpression predicts tumor recurrence of hepatocellular carcinoma after liver transplantation. Med Oncol 29:1810-1816. 10.1007/s12032-011-0004-Z

Langfelder P, and Horvath S. 2008. WGCNA: an R package for weighted correlation network analysis. BMC Bioinformatics 9:559. 10.1186/1471-2105-9-559

Law CW, Alhamdoosh M, Su S, Dong X, Tian L, Smyth GK, and Ritchie ME. 2016. RNA-seq analysis is easy as 1-2-3 with limma, Glimma and edgeR. F1000Res 5. 10.12688/f1000research.9005.3

Lee JG, Kang CM, Park JS, Kim KS, Yoon DS, Choi JS, Lee WJ, and Kim BR. 2006. The actual five-year survival rate of hepatocellular carcinoma patients after curative resection. Yonsei Med J 47:105-112. 10.3349/ymj.2006.47.1.105

Li X, Li B, Ran P, and Wang L. 2018. Identification of ceRNA network based on a RNA-seq shows prognostic lncRNA biomarkers in human lung adenocarcinoma. Oncol Lett 16:5697-5708. 10.3892/ol.2018.9336

Liu Z, Yan HY, Xia SY, Zhang C, and Xiu YC. 2016. Downregulation of long non-coding RNA TRIM52-AS1 functions as a tumor suppressor in renal cell carcinoma. Mol Med Rep 13:3206-3212. 10.3892/mmr.2016.4908

Loher P, and Rigoutsos I. 2012. Interactive exploration of RNA22 microRNA target predictions. Bioinformatics 28:3322-3323. 10.1093/bioinformatics/bts615

Lu QC, Rui ZH, Guo ZL, Xie W, Shan S, and Ren T. 2018a. LncRNA-DANCR contributes to lung adenocarcinoma progression by sponging miR-496 to modulate mTOR expression. $\mathrm{J}$ Cell Mol Med 22:1527-1537. 10.1111/jcmm.13420

Lu Y, Hu Z, Mangala LS, Stine ZE, Hu X, Jiang D, Xiang Y, Zhang Y, Pradeep S, RodriguezAguayo C, Lopez-Berestein G, DeMarzo AM, Sood AK, Zhang L, and Dang CV. 2018b. MYC Targeted Long Noncoding RNA DANCR Promotes Cancer in Part by Reducing p21 Levels. Cancer Research 78:64-74. 10.1158/0008-5472.CAN-17-0815 
306

307

308

309

310

311

312

313

314

315

316

317

318

319

320

321

322

323

324

325

326

327

328

329

330

331

332

333

334

335

336

337

338

339

340

341

342

343

344

345

346

Mao Z, Li H, Du B, Cui K, Xing Y, Zhao X, and Zai S. 2017. LncRNA DANCR promotes migration and invasion through suppression of lncRNA-LET in gastric cancer cells. Biosci Rep 37. 10.1042/BSR20171070

Maza E. 2016. In Papyro Comparison of TMM (edgeR), RLE (DESeq2), and MRN Normalization Methods for a Simple Two-Conditions-Without-Replicates RNA-Seq Experimental Design. Front Genet 7:164. 10.3389/fgene.2016.00164

Nishida N, and Fukuda Y. 2001. [Tumor suppressor RB gene and its related molecules in hepatocellular carcinoma]. Nihon Rinsho 59 Suppl 6:134-137.

Ou L, Wang D, Zhang H, Yu Q, and Hua F. 2018. Decreased Expression of miR-138-5p by lncRNA H19 in Cervical Cancer Promotes Tumor Proliferation. Oncol Res 26:401-410. 10.3727/096504017X15017209042610

Peng Q, Lao X, Chen Z, Lai H, Deng Y, Wang J, Mo C, Sui J, Wu J, Zhai L, Yang S, Qin X, and Li S. 2013. TP53 and MDM2 gene polymorphisms, gene-gene interaction, and hepatocellular carcinoma risk: evidence from an updated meta-analysis. PLoS One 8:e82773. 10.1371/journal.pone.0082773

Reimers M, and Carey VJ. 2006. Bioconductor: an open source framework for bioinformatics and computational biology. Methods Enzymol 411:119-134. 10.1016/S00766879(06)11008-3

Selcuk H. 2017. Prognostic Factors and Staging Systems in Hepatocellular Carcinoma. Exp Clin Transplant 15:45-49. 10.6002/ect.TOND16.L11

Sha S, Yuan D, Liu Y, Han B, and Zhong N. 2017. Targeting long non-coding RNA DANCR inhibits triple negative breast cancer progression. Biol Open 6:1310-1316. 10.1242/bio.023135

Shao M, and Li W. 2019. Transcriptional factor regulation network and competitive endogenous RNA (ceRNA) network determining response of esophageal squamous cell carcinomas to neoadjuvant chemoradiotherapy. PeerJ 7:e6668. 10.7717/peerj.6668

Siegel RL, Miller KD, and Jemal A. 2019. Cancer statistics, 2019. CA Cancer J Clin 69:7-34. $10.3322 /$ caac. 21551

Su P, Wang F, Qi B, Wang T, and Zhang S. 2017. P53 Regulation-Association Long NonCoding RNA (LncRNA PRAL) Inhibits Cell Proliferation by Regulation of P53 in Human Lung Cancer. Med Sci Monit 23:1751-1758.

Szklarczyk D, Morris JH, Cook H, Kuhn M, Wyder S, Simonovic M, Santos A, Doncheva NT, Roth A, Bork P, Jensen LJ, and von Mering C. 2017. The STRING database in 2017: quality-controlled protein-protein association networks, made broadly accessible. Nucleic Acids Res 45:D362-D368. 10.1093/nar/gkw937

Tellapuri S, Sutphin PD, Beg MS, Singal AG, and Kalva SP. 2018. Staging systems of hepatocellular carcinoma: A review. Indian J Gastroenterol 37:481-491. 10.1007/s12664018-0915-0

Valasek LS, Zeman J, Wagner S, Beznoskova P, Pavlikova Z, Mohammad MP, Hronova V, Herrmannova A, Hashem Y, and Gunisova S. 2017. Embraced by eIF3: structural and functional insights into the roles of eIF3 across the translation cycle. Nucleic Acids Res 
45:10948-10968. 10.1093/nar/gkx805

Varet H, Brillet-Gueguen L, Coppee JY, and Dillies MA. 2016. SARTools: A DESeq2- and EdgeR-Based R Pipeline for Comprehensive Differential Analysis of RNA-Seq Data. PLoS One 11:e0157022. 10.1371/journal.pone.0157022

Wang H, Liu W, Liu L, Wu C, Wu W, Zheng J, Zhang M, Chen X, Zhou B, Gao Z, and Huang J. 2017. Overexpression of centromere protein K (CENP-K) gene in hepatocellular carcinoma promote cell proliferation by activating AKT/TP53 signal pathway. Oncotarget 8:73994-74005. 10.18632/oncotarget.18172

Wang Y, He L, Du Y, Zhu P, Huang G, Luo J, Yan X, Ye B, Li C, Xia P, Zhang G, Tian Y, Chen R, and Fan Z. 2015. The long noncoding RNA lncTCF7 promotes self-renewal of human liver cancer stem cells through activation of Wnt signaling. Cell Stem Cell 16:413-425. 10.1016/j.stem.2015.03.003

Wang Y, Xue D, Li Y, Pan X, Zhang X, Kuang B, Zhou M, Li X, Xiong W, Li G, Zeng Z, and Yang T. 2016. The Long Noncoding RNA MALAT-1 is A Novel Biomarker in Various Cancers: A Meta-analysis Based on the GEO Database and Literature. J Cancer 7:9911001. 10.7150/jca.14663

Weng H, Huang H, Wu H, Qin X, Zhao BS, Dong L, Shi H, Skibbe J, Shen C, Hu C, Sheng Y, Wang Y, Wunderlich M, Zhang B, Dore LC, Su R, Deng X, Ferchen K, Li C, Sun M, Lu Z, Jiang X, Marcucci G, Mulloy JC, Yang J, Qian Z, Wei M, He C, and Chen J. 2018. METTL14 Inhibits Hematopoietic Stem/Progenitor Differentiation and Promotes Leukemogenesis via mRNA m(6)A Modification. Cell Stem Cell 22:191-205 e199. 10.1016/j.stem.2017.11.016

$\mathrm{Xu}$ D, Yu J, Gao G, Lu G, Zhang Y, and Ma P. 2018. LncRNA DANCR functions as a competing endogenous RNA to regulate RAB1A expression by sponging miR-634 in glioma. Biosci Rep 38. 10.1042/BSR20171664

Yao J, Shen X, Li H, Xu J, Shao S, Huang JX, and Lin M. 2018. LncRNA-ECM is overexpressed in esophageal squamous cell carcinoma and promotes tumor metastasis. Oncol Lett 16:3935-3942. 10.3892/ol.2018.9130

Yin L, Cai Z, Zhu B, and Xu C. 2018. Identification of Key Pathways and Genes in the Dynamic Progression of HCC Based on WGCNA. Genes (Basel) 9. 10.3390/genes 9020092

Yuan SX, Wang J, Yang F, Tao QF, Zhang J, Wang LL, Yang Y, Liu H, Wang ZG, Xu QG, Fan J, Liu L, Sun SH, and Zhou WP. 2016. Long noncoding RNA DANCR increases stemness features of hepatocellular carcinoma by derepression of CTNNB1. Hepatology 63:499-511. 10.1002/hep.27893

Zhang JC, Gao B, Yu ZT, Liu XB, Lu J, Xie F, Luo HJ, and Li HP. 2014. Promoter hypermethylation of p14 (ARF), RB, and INK4 gene family in hepatocellular carcinoma with hepatitis B virus infection. Tumour Biol 35:2795-2802. 10.1007/s13277-013-1372-0

Zhang Y, Wu SS, Chen XH, Tang ZH, Yu YS, and Zang GQ. 2017. Tripartite Motif Containing 52 (TRIM52) Promotes Cell Proliferation in Hepatitis B Virus-Associated Hepatocellular Carcinoma. Med Sci Monit 23:5202-5210.

Zhussupbekova S, Sinha R, Kuo P, Lambert PF, Frazer IH, and Tuong ZK. 2016. A Mouse 
Model of Hyperproliferative Human Epithelium Validated by Keratin Profiling Shows an Aberrant Cytoskeletal Response to Injury. EBioMedicine 9:314-323. 
Figure 1

Fig 1. Different expression of mRNA, miRNA and IncRNA levels between tumor and normal samples.

(A) Heatmap showing 2763 different expression of mRNA between tumor and normal samples in LIHC. (B) Volcano map for 310 different expression miRNA. Dots in red and green indicate high and low expression of miRNA in cancer, respectively. (C) Volcano map for 1962 different LncRNA. Dots in red and green indicate high and low expression of IncRNA in cancer, respectively.

A

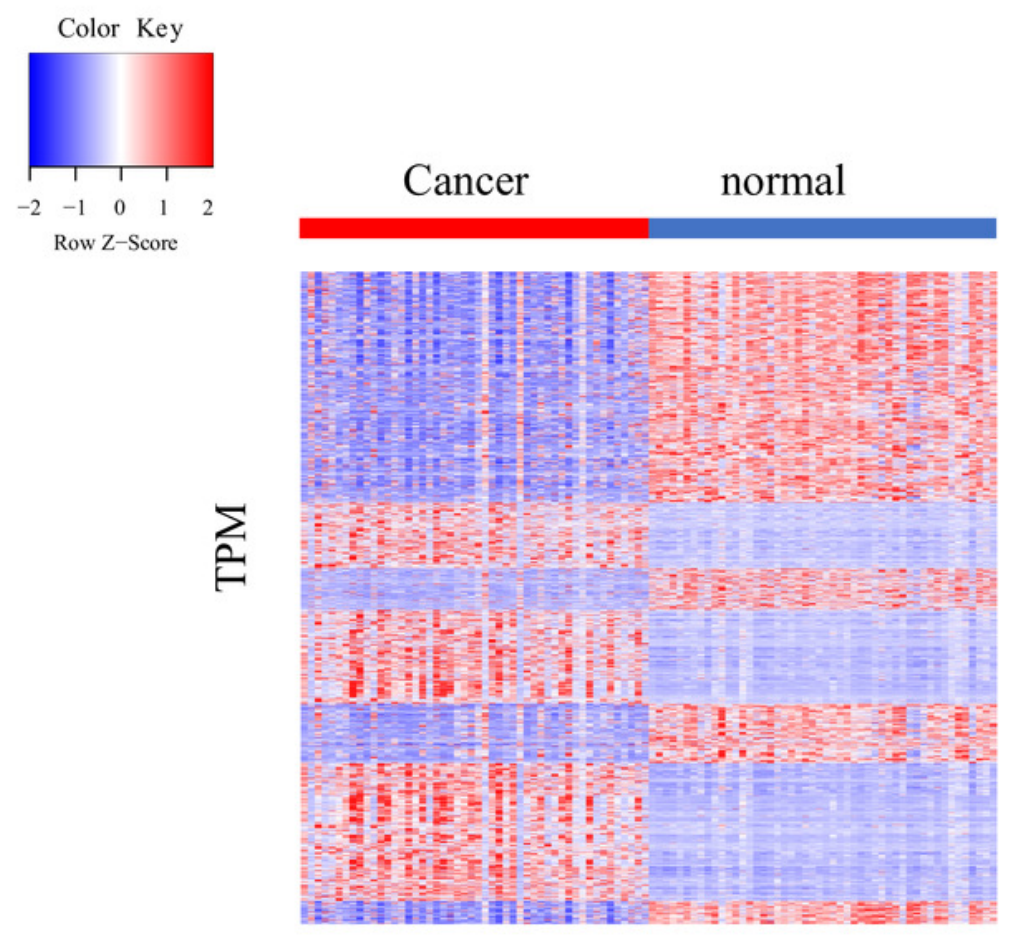

$\mathrm{B}$

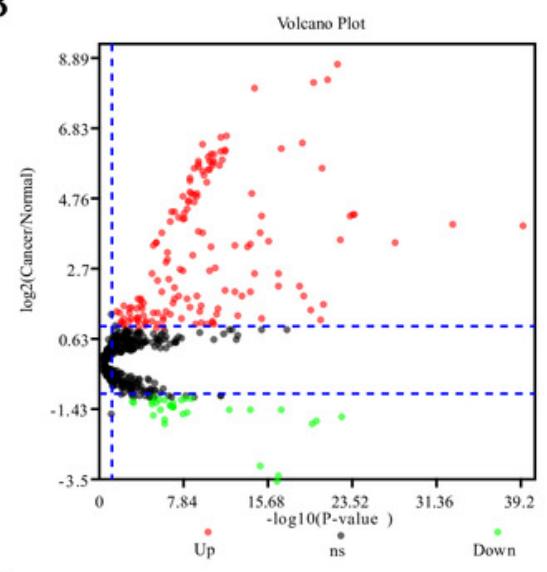

$\mathrm{C}$

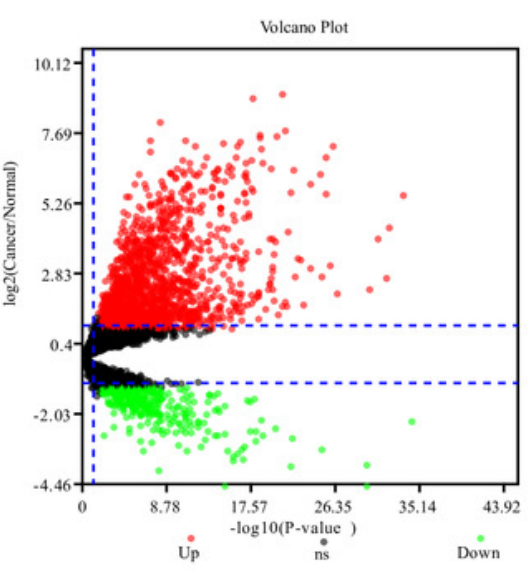


Figure 2

Fig 2. Results of Weight Gene Co-expression Network Analysis (WGCNA).

(A) shows the clustering dendrogram of co-expression IncRNA based on topological overlap.

(B) Module-TNM stage correlative analysis. Each row corresponds to a module eigengene, each column corresponds to a TNM stage. Heatmap block with p-values and correlation coefficient. The red box in the figure shows the module with higher correlation coefficient in the three stages of TNM. The blue box in the figure shows the module with negative correlation coefficient in the three stages of TNM.

A

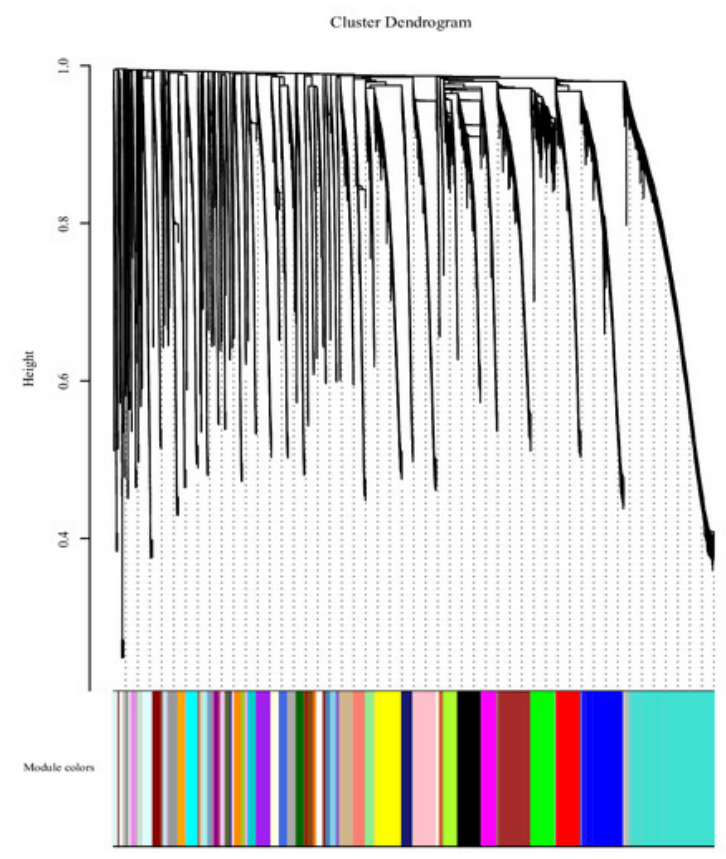

$\mathrm{B}$

Module-trait relationships

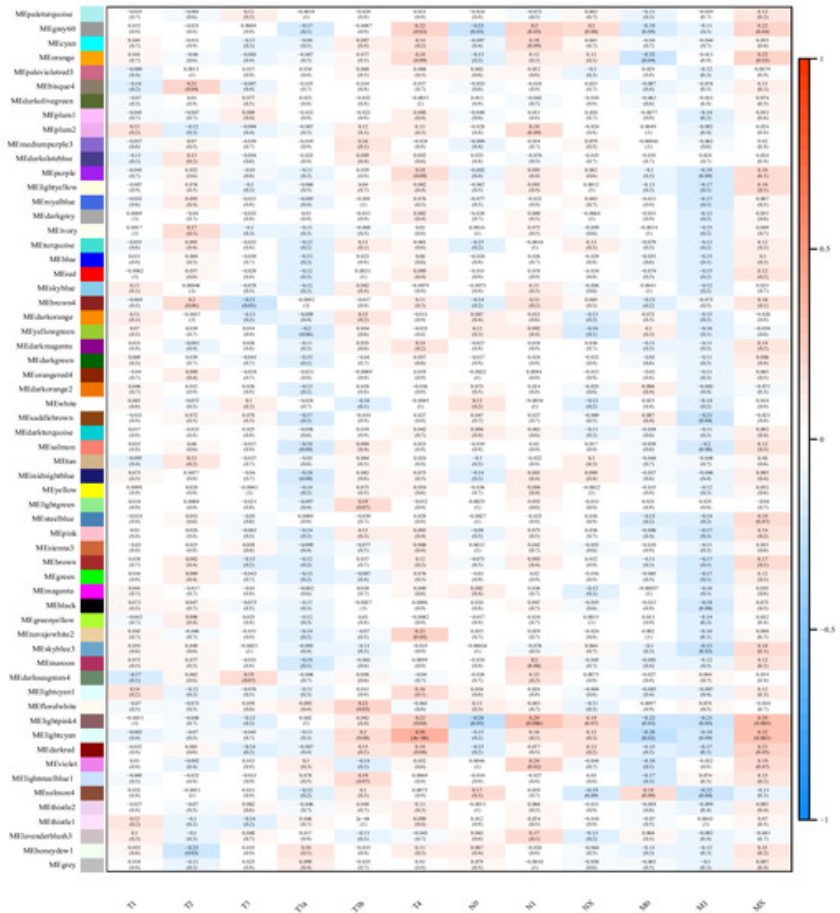


Figure 3

Fig 3. CeRNA network in LIHC and survival curves.

(A) Rosered dots represent overlaping IncRNA selected from co-expression analysis and RNA22 which can identify microRNA binding sites. The bigger the dots, the higher the degree value, the more important nodes in the network. (B) Survival curve of patients with high expression of DANCR and low expression of DANCR. Survival curve of patients with high expression of AL671710.1 and low expression of AL671710.1.

A

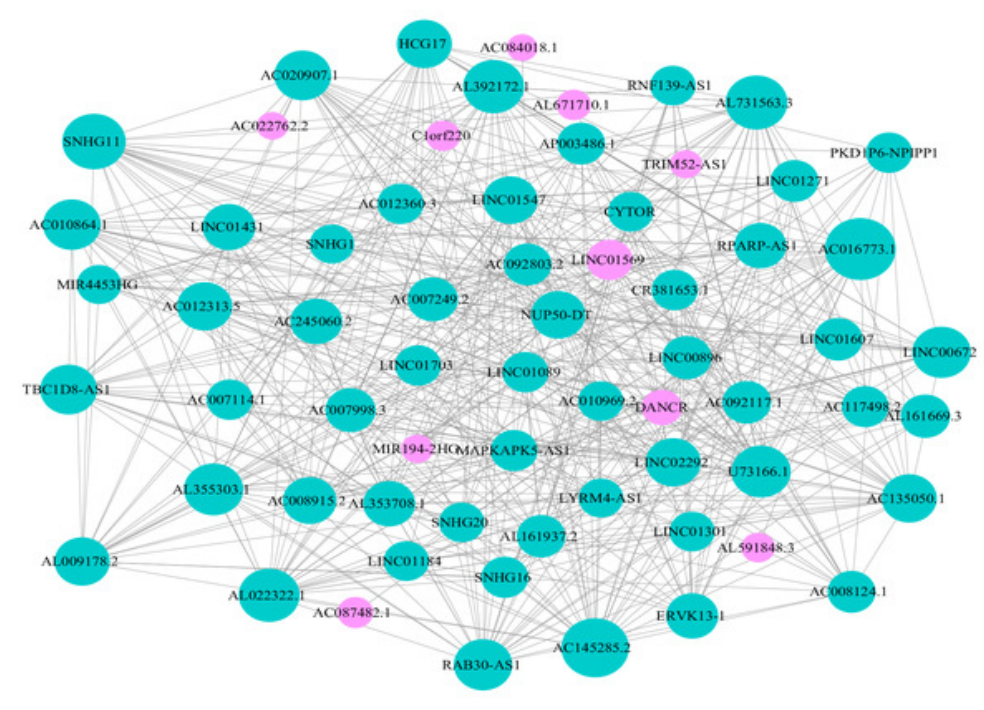

B
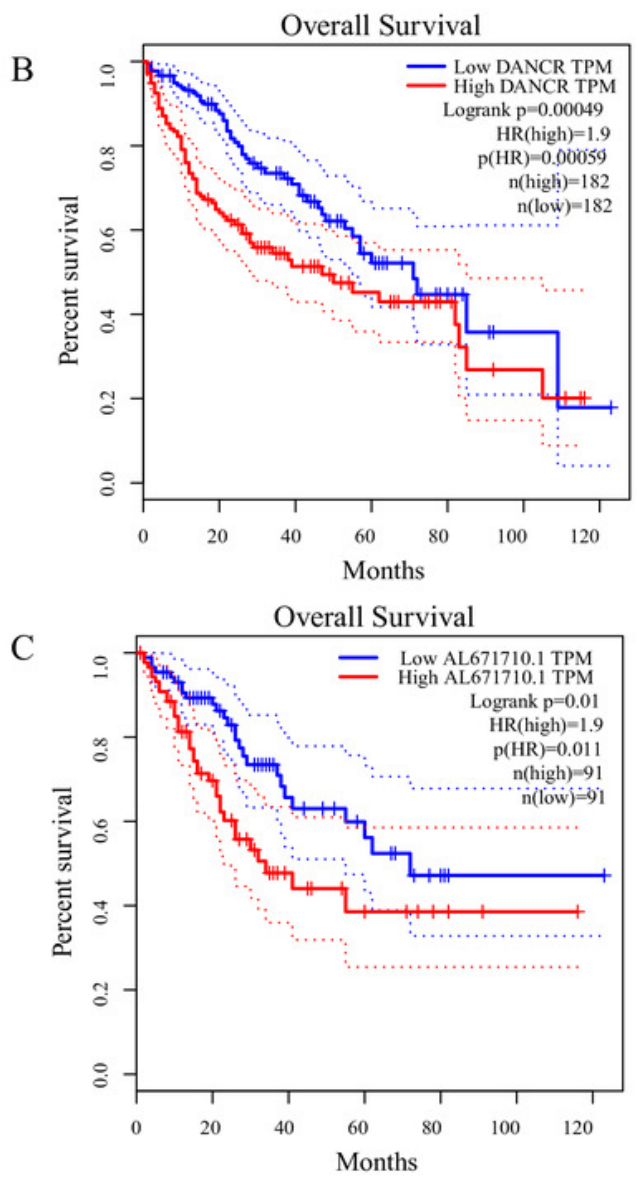


\section{Figure 4}

Fig 4. Interaction of eight IncRNA and target mRNA.

The dots in the arrow shape represent the IncRNA, and red wine color dots represent the mRNA in the figure. It can be seen that the eight important IncRNA AL6717.1, TRIM52-AS1, C1220C1, DANCR, LINC01569, AC084018.1 participated in many regulation of mRNA.

A

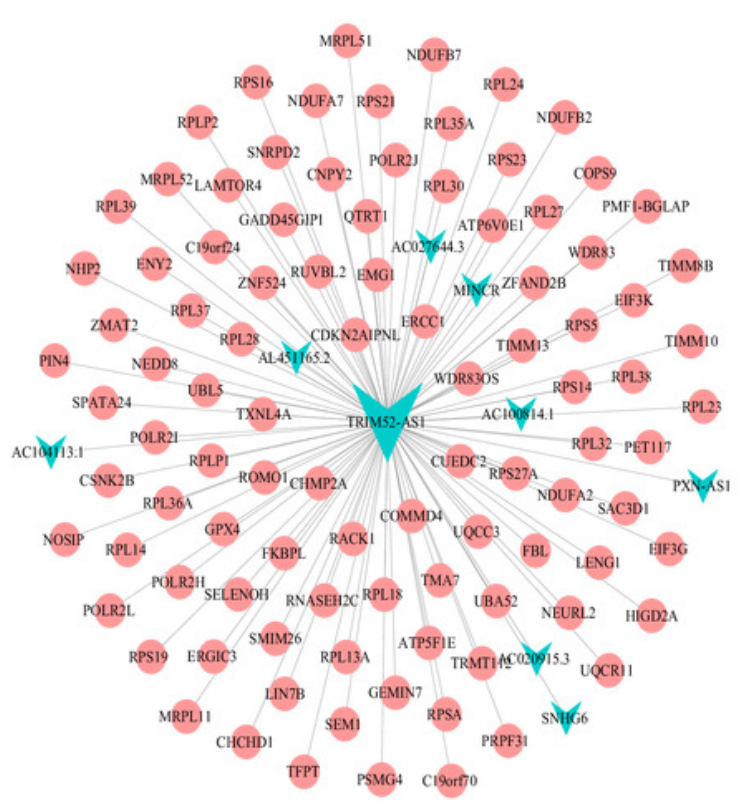

B

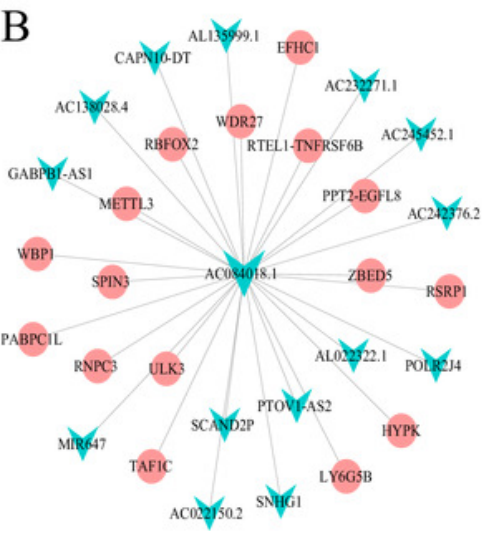

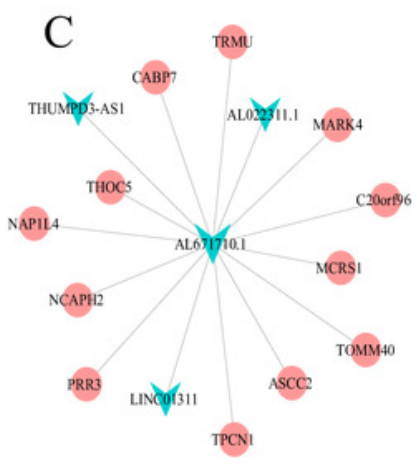

F

RADSPASI

E

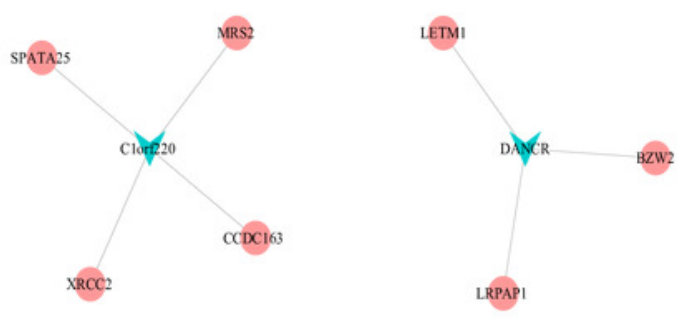

G

एUT

undopists

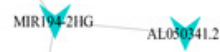

Acolig/sis.1
$\mathrm{H}$ ApDody 13.1 Acolesfor2 Acoupo 62 
Figure 5

Fig 5. GO and KEGG pathway enrichment analysis of target gene.

(A) GO enrichment of mRNA interact with IncRNA. Points of different shapes represent BP, CC and MF of GO, and the size of points represents the number of gene enriched in the $\mathrm{GO}$ function, the number of different colors represents $P$ value. (B) KEGG pathway is enriched in Ribosome and huntington disease.

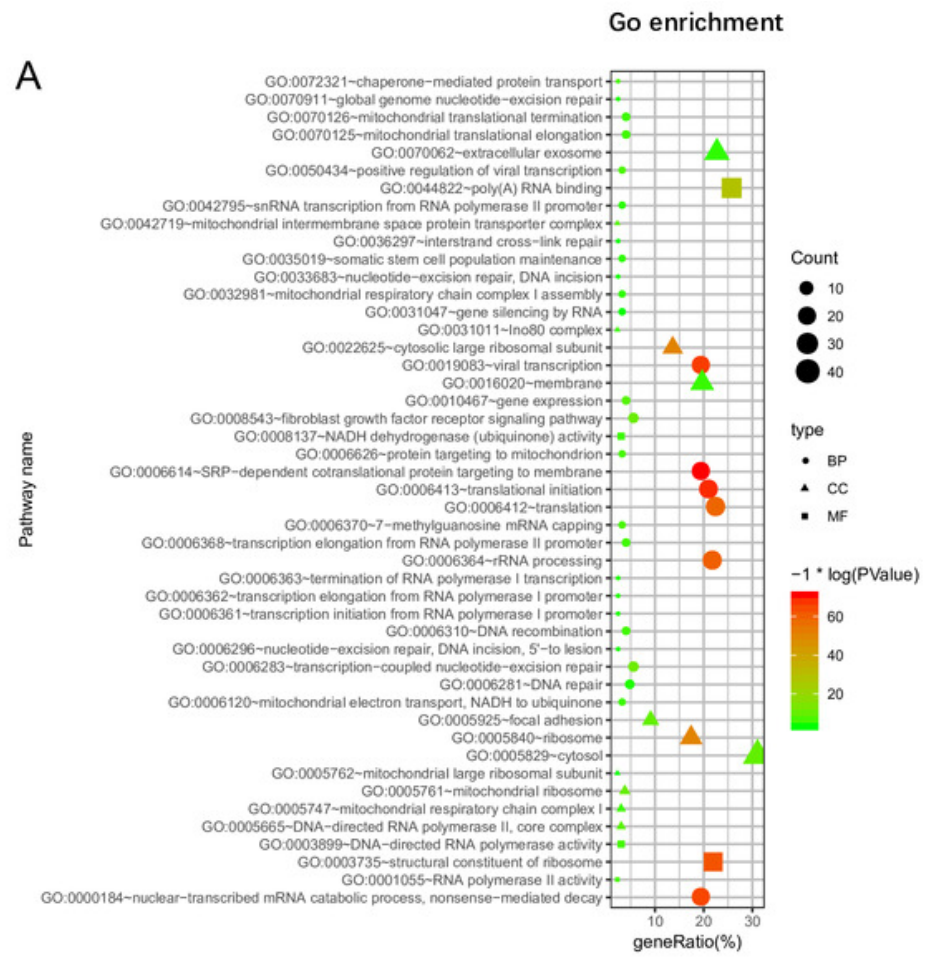

B

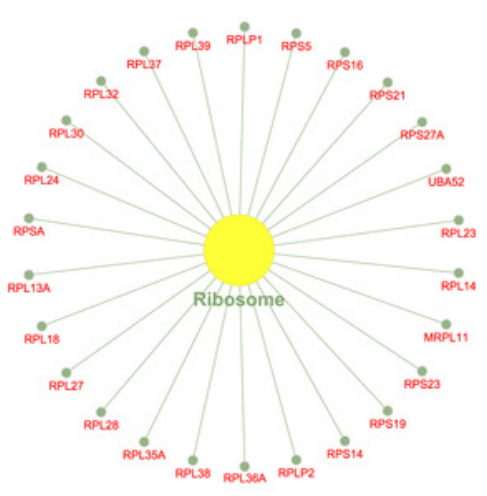

C

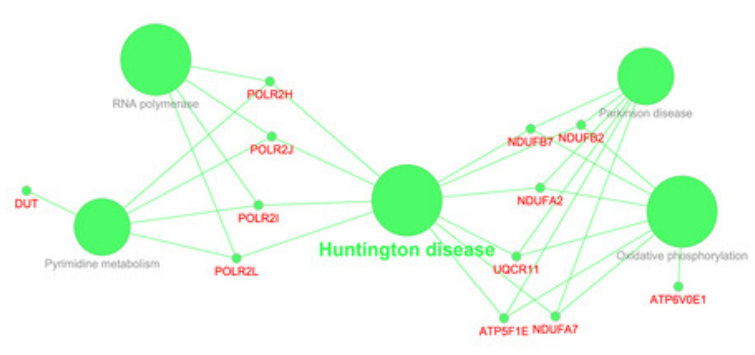


Figure 6

Fig 6. The protein-protein interactions of RNA and conclusion summary.

(A) The PPI network of RNA with degree bigger than 10 in string database. The larger the dots in the graph, the more interaction between the RNA corresponding proteins and other proteins. (B) Conclusion summary.

A

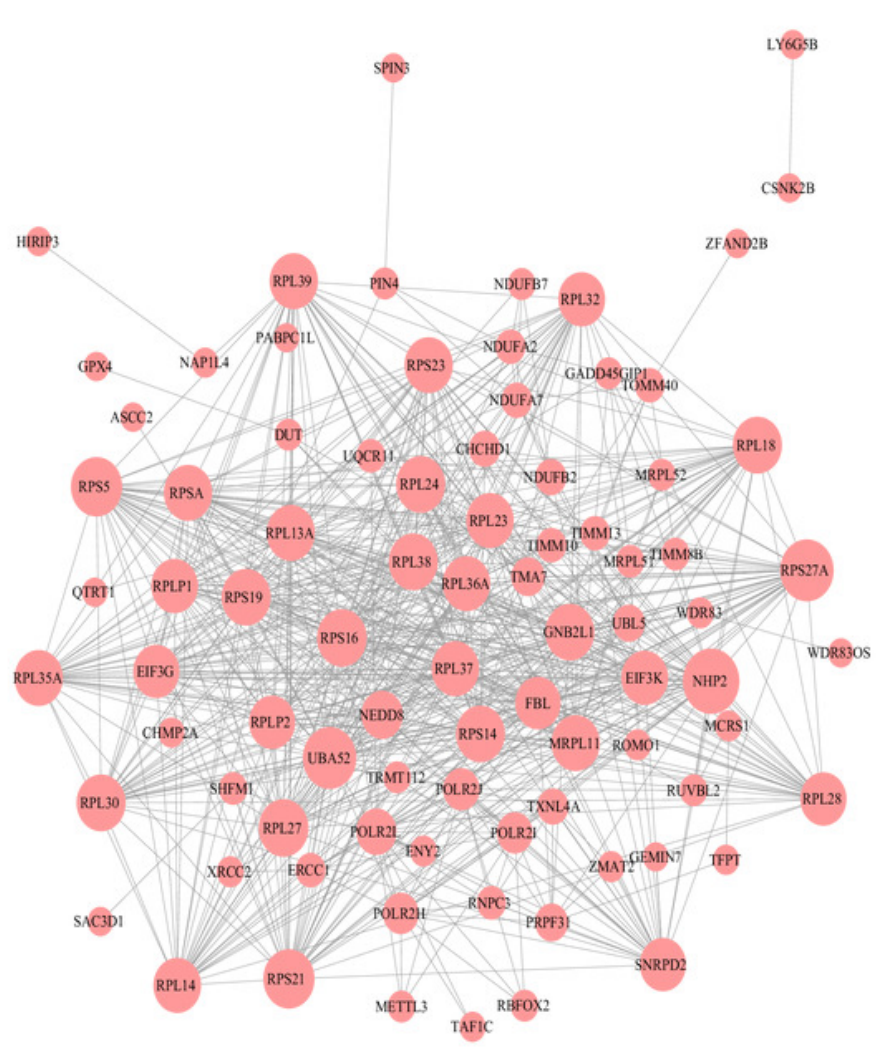

$\mathrm{B}$

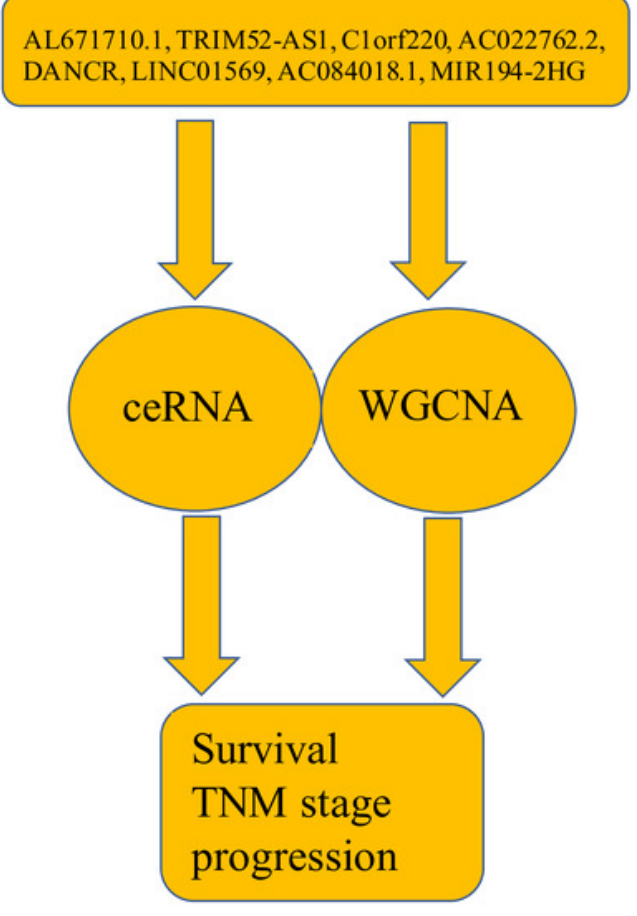

